\title{
Promoción de medidas higiénico-dietéticas para el manejo del SOP, por el profesional de enfermería.
}

\section{Promotion of Hygienic-dietary actions to manage SOP, for nurse professional}

\begin{abstract}
Cinthia Sarahi Gabriel-Hernandez ${ }^{a}$, Fernando Ochoa-cortes ${ }^{b}$
Abstract:

Polycystic ovary syndrome is a metabolic endocrine disorder, affecting women during reproductive age. According to the Rotterdam diagnostic criteria patients must present two out of three: clinical or biochemical hyperandrogenism, oligo/anovulation or polycystic ovaries. The nurse plays an important role in the polycystic ovary syndrome, by promoting modification of people's lifestyles, through physical activity, changes on feedings behaviours and through interventions that help to prevent or reduce the alterations associated with PCOS. Insulin resistance, menstrual disorders, obesity and hirsutism are the main reasons for seeking medical advice, thus hygienic-dietary actions implementation is key to reduce the aforementioned alterations through promotion, implementation and follow up of these actions as a nurse intervention.
\end{abstract}

\section{Keywords:}

Polycystic ovary syndrome, lifestyles, hirsutism, insulin resistance, hyperandrogenism

\section{Resumen:}

El síndrome de ovario poliquístico es una alteración endocrino metabólica que afecta a mujeres durante la edad reproductiva. De acuerdo a los criterios diagnósticos de Rotterdam los pacientes deben presentar dos de los siguientes tres; hiperandrogenismo clínico o bioquímico, oligo/anovulación u ovarios poliquísticos. La enfermería desempeña un rol importante en el síndrome de ovario poliquístico, al promover la modificación del estilo de vida de la persona a través de actividad física, cambios en las conductas alimentarias y por medio de intervenciones que ayuden a prevenir o reducir alteraciones asociados al SOP. La resistencia a la insulina, trastornos menstruales, obesidad e hirsutismo son los principales motivos de consulta, debido a eso es imperante implementar medidas higiénico-dietéticas con el propósito de disminuir las alteraciones ya mencionadas por medio de la promoción, implementación y seguimiento de estas acciones como una intervención de enfermería.

\section{Palabras Clave:}

Síndrome del ovario poliquístico, estilos de vida, hirsutismo, resistencia a la insulina, hiperandrogenismo

\section{Introducción}

En el año de 1935, Stein y Leventhal llamaron síndrome de Stein Leventhal al cuadro clínico compuesto por pequeños quistes en el ovario, amenorrea, obesidad e hirsutismo y conocido en la actualidad como síndrome de ovario poliquístico (SOP). EL SOP representa un problema de salud y es la causa más común de problemas de fertilidad afectando entre un $6-10 \%$ de mujeres mexicanas en edad reproductiva. El síndrome está definido como una condición heterogénea y cuyos criterios fueron definidos en el consenso de Rotterdam en mayo del 2003 (Chiliquinga Villacis et al. 2017). La etiología es multifactorial y se asocia a diversos factores predisponentes como: la menarca temprana, familia con antecedentes de la enfermedad, aumento del IMC a inicios de menarca, además la resistencia a la insulina presenta un riesgo debido a las dislipidemias y diabetes mellitus. En muchos casos, la modificación de los estilos de vida en mujeres con esta patología llega a lograr disminuir los problemas relacionados, como: disminución del IMC, alteraciones reproductivas y

Autor de Correspondencia, Universidad Autónoma del Estado de Hidalgo, https://orcid.org/0000-0002-3600-3372, Email: ga392812@uaeh.edu.mx

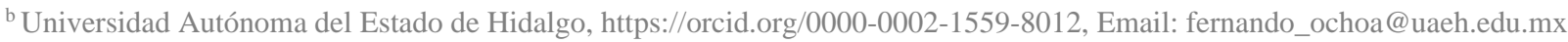


reducir el exceso de andrógenos. Sin embargo, la implementación de estilos de vida saludables a largo plazo, suelen ser difíciles de llevar, por lo que se recomienda el acompañamiento profesional de las medidas higiénico dietéticas como un tratamiento de primera línea contra el SOP, basados en programas, planes de cuidados enfermeros, además de la implementación de actividades enfocadas en la disminución del peso corporal, apoyo psicológico, actividad física y cambios en la dieta; con el fin de mejorar el estado físico, mental y social del individuo.

\section{Síndrome de ovario poliquístico}

El síndrome de ovario poliquístico (SOP) es un desorden endocrino, que afecta a las mujeres en la edad reproductiva, además de ser una de las principales causas de infertilidad. La prevalencia del SOP es de 5$8 \%$ tanto de mujeres adolescentes como adultas (Moncada, Ramos, and Gerardo Muñoz 2018). En la mayoría de los casos las mujeres presentan sobrepeso $\mathrm{u}$ obesidad asociado a la resistencia a la insulina, llegando a complicar aún más los problemas reproductivos y metabólicos. La etiología del SOP es multifactorial e incluye los siguientes factores predisponentes; menarca temprana, aumento del IMC a inicio de menarca y factores genéticos; en la mayoría de los casos, las hijas de mujeres con SOP presentan síntomas a temprana edad además niveles elevados de andrógenos (testosterona) y resistencia a la insulina. Se considera que el SOP es un círculo vicioso, debido a que la obesidad puede genera resistencia a la insulina además aumentar el riesgo de dislipidemias, diabetes mellitus tipo 2, entre otros (Balestrini 2016).

Una detección rápida de los síntomas ayuda a prevenir complicaciones ocasionadas por el SOP, entre los síntomas más comunes encontramos (González et al. 2010);

- Acné

- Alopecia (perdida de pelo)

- Obesidad

- Trastornos menstruales

- Hirsutismo (aparición de vello en tórax, cara, espalda, muslos y abdomen).

De acuerdo a diferentes estudios (Alicia et al. 2017), los principales motivos de consulta médica en mujeres con esta patología son la infertilidad y el aumento del IMC En cuanto a la descripción del SOP se ha modificado con el pasar de los años; a partir del año 1935 se consideraba la presencia de amenorrea, hirsutismo y obesidad como características del SOP, en 2003 se establecieron los criterios de Rotterdam que consisten en: hiperandrogenismo clínico (presencia de acné, alopecia e hirsutismo) o bioquímico (niveles de testosterona total e índice androgénico libre), oligo/anovulación, alteraciones ecográficas (12 o más folículos menores de $10 \mathrm{~mm}$, aumento de volumen ovárico mayor a $10 \mathrm{ml}$.) (Moncada, Ramos, and Gerardo Muñoz 2018). Sin embargo, las mujeres con el síndrome no suelen presentar todos los criterios, estos varían de acuerdo a cada persona, estableciéndose 4 fenotipos, (véase en tabla 1). El fenotipo 1 o clásico, es el más común y presente en la mayoría de los casos; para considerarse síndrome de ovario poliquístico la persona debe de presentar 2 o más de los criterios ya mencionados: Por otra parte, se debe brindar información sobre el SOP, los problemas que traerá a futuro, así como los posibles riesgos que implique (Orrego 2019).

\section{Prevalencia}

De acuerdo a distintos reportes, existe cierta variabilidad en la prevalencia y depende de los criterios diagnósticos y la población que se desea abarcar. A nivel mundial el SOP afecta a 5 de cada 10 mujeres $y$ en América Latina se presenta una prevalencia del 10-30\% (Poliqu 2019). Por otro lado, se reporta diferencias en la prevalencia según su raza, alrededor $8.0 \%$ de las mujeres de raza negra, 5.6 en asiáticas, $4.8 \%$ en raza blanca y $13 \%$ en méxicoamericanas (Carmona-Ruiz et al. 2015). Así mismo, mujeres mexicanas presentan entre 14.8-29.3\% de prevalencia de SOP según los criterios usados para su diagnóstico, reportando el fenotípico clásico como el más frecuente, seguido del 2 y 4.(Carmona-Ruiz et al. 2015). Un estudio realizado a nivel nacional en el año 2016 arrojo una incidencia de aproximadamente el 6\%, sin especificar el fenotipo (Poliqu 2019). En relación a los grupos étnicos existe mayor prevalencia en los mixtecos de Baja california $(26.2 \%)$ y los Yaquis de Sonora (18.3\%), a diferencia de los mazatecos con la menor incidencia de casos (2.0\%) (Esparza-Romero 2021).

\section{Diagnostico}

Las manifestaciones más perceptibles del síndrome de ovario poliquístico son, el acné, alopecia (perdida de pelo), obesidad, virilización (voz masculina, clitoromegalia, atrofia de mamas, hipertrofia muscular), alteraciones menstruales e hirsutismo (aparición de vello en tórax, cara, espalda, muslos, abdomen); además para el diagnóstico se emplean los siguientes criterios (Tabla 1). Otros formas de detección del SOP son por medio de pruebas de laboratorio, por ejemplo, niveles séricos de testosterona, hormona folículo estimulante, progesterona sérica y ultrasonido pélvico, con el fin de identificar alteraciones a nivel hormonal. También deben realizarse estudios para descartar otras posibles enfermedades (disfunción tiroidea, hiperplasia adrenal congénita, síndrome de Cushing, hiperprolactinemia y tumores secretores de andrógenos) que podrían entorpecer el diagnostico de SOP. De la misma forma se debe realizar un perfil de lípidos, glucosa sérica e insulina sérica, para evaluar posibles comorbilidades (González et al. 2010). En ocasiones en el SOP favorece la aparición de problemas como estrés, 
ansiedad, depresión, percepción negativa de la imagen corporal, por lo que suele confundirse y se atribuye a los trastornos menstruales inducidos por presencia de estrés, ansiedad, entre otras.

\section{Comorbilidades}

EI SOP es una enfermedad de suma importancia no solo por la complejidad del diagnóstico, sino por las comorbilidades asociadas a la enfermedad como son: obesidad, presente en muchos de los casos, suele originarse por factores genéticos, alimentarios, sedentarismo y falta de actividad física; diabetes mellitus tipo 2; resistencia a la insulina posee mayor incidencia en mujeres con sobrepeso y obesas a diferencia de las delgadas; hipertensión arterial (HTA); dislipidemias (DLP); apnea del sueño (la respiración se detiene para después comenzar a los pocos segundos); cáncer de endometrio; hiperplasia; infertilidad; la acantosis nigricans, presentándose en forma de manchas oscuras en la zona de la ingle, axilas, cuello, suele aparecer en personas con obesidad y diabetes; hiperprolactinemia; síndrome metabólico; entre otros. Debido a los posibles riesgos que pueden correr las mujeres con SOP es importante dirigir el tratamiento adecuado hacia cada uno (Vidilia et al. 2019).

\section{Tabla 1. Criterios diagnósticos del SOP}

Criterios de diagnostico
-Fenotipo 1 (clásico)
A. Hiperandrogenismo clínico o
bioquímico
B. Oligo/anovulación
C. Alteraciones ecográficas

\section{-Fenotipo 2}

A. Oligo/anovulación

B. Hiperandrogenismo

\section{-Fenotipo 3}
A. Hiperandrogenismo
clínico 0 bioquímico
B. Alteración ecográfica

\section{-Fenotipo 4 (Normo androgénico) \\ A. Alteración ecográfica \\ B. Oligo/anovulación}

Elaboración propia.

\section{Tratamiento}

El tratamiento está enfocado en lograr disminuir el sobrepeso u obesidad y las alteraciones metabólicas que presenta la mayoría de mujeres. El tratamiento es variado y dirigido a resolver los problemas específicos que afecten a la persona con SOP, la terapéutica del hirsutismo se divide en 2 tipos de tratamiento:

1. Farmacológico (antiandrógenos y anticonceptivos hormonales): suele ser el tratamiento de primera línea y empleados en periodos mayores de 6 meses, ya que disminuye los niveles de andrógenos a nivel ovárico y suprarrenal ocasionando mejoras en el aspecto físico y mental.

2. Cosmético: empleados para el retiro de pelo terminal (pelo más grueso y largo, suele aparecer en cualquier parte del cuerpo) mediante rasurado y depilación (cremas, pinzas).

El régimen terapéutico suele emplear agentes sensibilizadores de la insulina como, metformina y tiazolinedionas, favoreciendo el mejoramiento de la resistencia a la insulina y disminuyendo el hiperandrogenismo (Rivera, Rodríguez, and Guarneros 2017). La infertilidad durante SOP suele estar relacionada con alteraciones nutricionales vinculadas con los estilos de vida que lleva la persona, lo que conduce al sobrepeso u obesidad. El tratamiento de primera línea suele ser citrato de clomifeno, pero cuando esta falla, la segunda línea para inducir la ovulación son las gonadotropinas, el inconveniente es el embarazo múltiple y costo elevado. Como todo medicamento llega a generar efectos indeseables (Tabla 2) como en el caso de los hormonales, suele producir hipertrigliceridemia y evento trombótico, por otra parte, otros medicamentos generan hiperestimulación ovárica, dermatitis química, irritación y eritema (Alfaro Murillo, Salas Ramírez, and Zúñiga Fallas 2021).

\section{Sensibilizadores de la insulina}

La resistencia a la insulina, es tema importante en el SOP, ocurre cuando las células no responden de manera correcta a la insulina; de manera que no absorbe la glucosa obtenida de los alimentos, lo que conlleva al páncreas a producir más insulina para que la glucosa entre en la célula. Los sensibilizadores de insulina son fármacos con acción euglucémiante, es decir, ayudan disminuyendo la concentración de glucosa plasmática (Monteagudo Peña et al. 2019).

Entre estos medicamentos encontramos la metformina, responsable de regular los niveles de glucosa, favorece la disminución de peso y aunque su efecto de la restauración de la función ovulatoria no es tan efectivo como el citrato de clomifeno, se prefiere ya que, disminuye los riesgos de hiperestimulación ovárica y juega un rol importante en la reducción de abortos (González et al. 2010). La metformina es recomendada en personas con diabetes mellitus tipo 2, prediabetes y síndrome metabólico; promueve los ciclos menstruales y ovulación en mujeres con irregularidades menstruales. Utilizado en mujeres embarazadas con diabetes gestacional, disminuye el riesgo de aborto. Entre los efectos que puede generar su consumo son: nauseas, 
Tabla 2. Reacciones adversas y contraindicaciones de fármacos usados en SOP.

\begin{tabular}{|c|c|c|c|}
\hline Medicamento & Indicaciones & Reacciones adversas & Contraindicaciones \\
\hline $\begin{array}{l}\text { Citrato de } \\
\text { clomifeno }\end{array}$ & $\begin{array}{l}\text { Infertilidad en } \\
\text { hombres y mujeres }\end{array}$ & $\begin{array}{lr}\begin{array}{l}\text { Náusea, } \\
\text { hipertensión }\end{array} & \text { vómito, } \\
\text { cefalea, mareos, } & \\
\text { visión } & \text { borrosa, } \\
\text { escotomas, } & \text { fatiga, } \\
\text { inquietud, } & \\
\text { hipersensibilidad. }\end{array}$ & $\begin{array}{l}\text { Enfermedad hepática } \\
\text { Insuficiencia hepática } \\
\text { Tumores } \\
\text { Depresión mental } \\
\text { Tromboflebitis }\end{array}$ \\
\hline Espironolactona & $\begin{array}{l}\text { Hirsutismo } \\
\text { femenino. }\end{array}$ & $\begin{array}{l}\text { Hiperpotasemia, mareo, } \\
\text { eritema, ginecomastia. }\end{array}$ & Hiperpotasemia \\
\hline $\begin{array}{l}\text { Gonadotrofinas } \\
\text { postmenopáusicas } \\
\text { Humanas }\end{array}$ & Induce la ovulación & $\begin{array}{l}\text { Hiperestimulación } \\
\text { ovárica con } \\
\text { aumento de los ovarios } \\
\text { Embarazo } \\
\text { múltiple }\end{array}$ & $\begin{array}{lr}\text { Disfunción } & \text { tiroidea. } \\
\text { Lesión orgánica } \\
\text { intracraneal. } \\
\text { Hemorragias uterinas } \\
\text { de origen no } \\
\text { determinado }\end{array}$ \\
\hline
\end{tabular}

diarrea, dolor abdominal, parestesia y anemia (Velázquez 2016). La administración de metformina disminuye varios síntomas causados por el SOP, sin embargo, no es recomendable si el paciente presenta insuficiencia renal debido a que la metformina se elimina por esta vía (Rivera, Rodríguez, and Guarneros 2017).

\section{Estilos de vida saludable durante el SOP}

El tratamiento del SOP tiene como fin disminuir la morbilidad en personas que cursan con obesidad y alteraciones metabólicas basados en los cambios del estilo de vida, antiandrógenos y anticonceptivos orales, mejorando la salud de la persona a tal grado de poder llevar una vida normal, recordemos que el tratamiento debe ser individualizando basándose en los fenotipos del SOP, síntomas y posibles riesgos que pueda presentar. Es importante destacar el abandono de conductas como el consumo de tabaco, disminución del consumo de alcohol y recibir ayuda psicología mediante terapias.

Los "estilos de vida saludable" no solo consisten en dietas o disminuir las porciones, sino en la modificación de actitudes, hábitos y conductas que conduzcan a mejorar la calidad de vida. Algunos ejemplos son, realizar deporte (30 min diarios o 5 días a la semana), llevar una dieta baja en calorías 1200-1400 kcal/día y dejar de consumir sustancias psicoactivas como alcohol y tabaco; (la Gómez Cruz et al. 2016). En el caso de las dietas, se elaboran de acuerdo a las características y necesidades de cada persona, sustituyendo alimentos poco nutritivos por unos más sanos en la proporción requerida para el individuo. Los puntos ya mencionados son clave en el tratamiento del SOP, dado que se caracteriza por ser cíclico; el exceso de andrógenos aumenta la grasa abdominal, empeorando la resistencia a la insulina, incrementando el riesgo de padecer diabetes mellitus tipo 2, hipertensión arterial, problemas respiratorios, entre otros.

La promoción como una estrategia busca mejorar la calidad de vida de las personas mediante intervenciones de salud, así como mantenimiento físico (Calpa Pastas et al. 2019). Algunas recomendaciones de enfermería son:

- Preguntar si realiza actividad física y con qué frecuencia

- Brindar asesoramiento dietético en personas con factores de riesgo cardiovascular y obesidad

- Ofrecer información acerca de los problemas que implica fumar, así como ofrecer ayuda para dejarlo

- Brindar información sobre los riesgos y beneficios de los estilos de vida

Poner en práctica la modificación de los estilos de vida, como la dieta y el ejercicio, ayuda a mejorar los síntomas y reducir el estrés. Las mujeres con esta patología suelen ser más susceptibles a cambios emocionales debido a las alteraciones que se producen en su cuerpo, motivo por el cual el profesional de enfermería debe atender esa problemática mediante actividades que ayuden a salir adelante al paciente (Suarez Coba et al. 2019). Los estilos de vida saludable logran disminuir los problemas relacionados con el SOP, mejoran la ovulación, mantienen un peso adecuado, disminuyen los niveles de colesterol y presión arterial. Aunque aún se duda de la relación entre el consumo de alcohol y tabaco con el SOP; el alcohol posee un alto contenido energético, aumentando el apetito provocando aún más su consumo, lo que conlleva a la disminución de la oxidación de lípidos (grasas), contribuyendo al aumento de peso corporal y producir sobrepeso $u$ obesidad (Luis Higuera-Sainz et al. 2017). El tabaquismo está relacionado con la aparición de enfermedades respiratorias y cardiovasculares y se encuentra asociado al aumento de colesterol plasmático total. La modificación de los estilos de vida se considera factor clave en el tratamiento de las mujeres con SOP así como la prevención de otras posibles enfermedades. Los planes de cuidados enfermeros tienen como fin aplicar cuidados mediante evidencia científica que 
ayude a resolver los problemas que ocasiona el SOP en la mujer, y por medio de los cambios del estilo de vida disminuir el tejido adiposo, además se logra disminuir las alteraciones reproductivas, el exceso de andrógenos, reducir la cantidad de quistes ováricos, restaurar ciclos menstruales, aminorar los riesgos de problemas cardiovasculares y comenzar a tener una mejor ovulación (Huérfano and Ortiz 2016). Se demostró que después de 6 meses de cambio en la dieta de 12001400 kcal-día, en el $36.9 \%$ de las mujeres desaparecieron los síntomas, y en $47.7 \%$ desaparecieron parcialmente; lo anterior independientemente del nivel de peso perdido (Huérfano and Ortiz 2016). Lo anterior ha dado origen a ensayos clínicos de 12 meses para elucidar los efectos positivos de la implementación multidisciplinaria de una terapia Cognitiva-Comportamiento-Estilo de vida (CBP, por sus siglas en inglés) en el SOP, ya que una disminución del $5-10 \%$ en peso corporal pueden ser suficientes para mejorar los síntomas del SOP. Además de que esta terapia mejora significativamente el estado emocional de las pacientes (G. Jiskoot et al. 2017).

La enfermería en promoción de estilos de vida saludable está encaminada a prevenir o parar el avance de una enfermedad además describe los hábitos alimenticios que se deben llevar, así como la actividad física; por otra parte, se encarga de educar a la población sobre hábitos insanos, fortaleciendo la prevención y control de enfermedades. Sin embargo, los estilos de vida saludable a largo plazo suelen ser difíciles de mantener, de tal manera que se emplean sensibilizadores de insulina en conjunto con medidas higiénico dietéticas, estableciéndose como un tratamiento importante del SOP.

\section{Las medidas higiénico dietéticas como una alternativa para el SOP}

Las medidas higiénico dietéticas son un conjunto de acciones dirigidas a disminuir la grasa abdominal, disminuir los niveles de andrógenos, mejorar la alteraciones menstruales y reproductivas entre otras. El tratamiento del SOP debe iniciar con las medidas higiénico-dietéticas que comprenden: la disminución del peso corporal en personas con sobrepeso u obesidad, mediante la actividad física, una dieta baja en calorías, disminución del ambiente obesogenico, la utilización de sensibilizadores de insulina (metformina), el abandono hábitos nocivos como el consumo de alcohol y tabaco, además de brindar apoyo psicológico; los planes de cuidados enfermeros brindan la facilidad para atender estas problemáticas que se llegan a presentar (Tabla.5). Algunas recomendaciones de enfermeria son (secreraria de salud 2016):

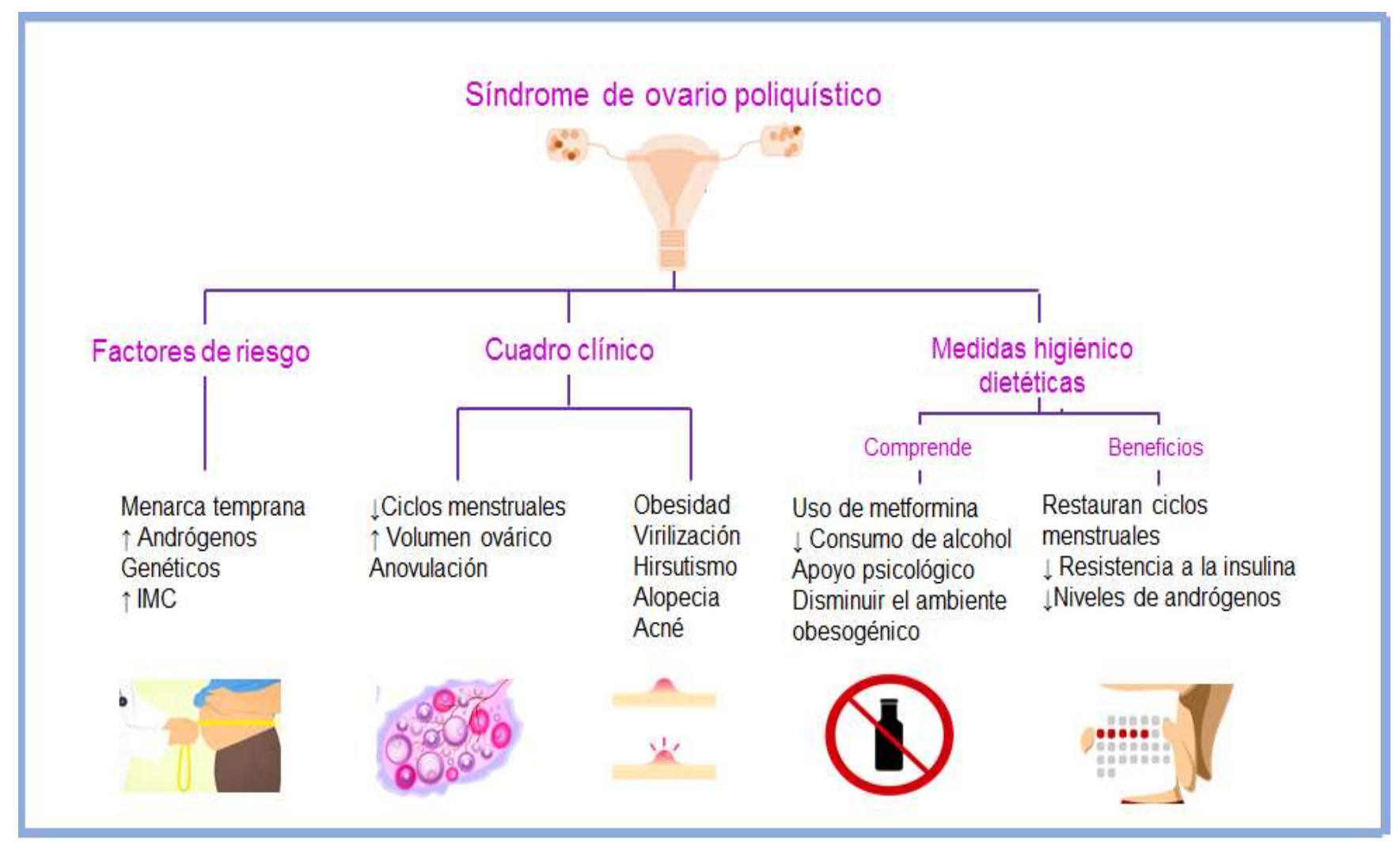

Figura 1. El síndrome de ovario poliquístico es un desorden endocrino de origen multifactorial evidenciado por el aumento del IMC, menarca temprana y aumento de los niveles de andrógenos; suele ocasionar anovulación trayendo consigo problemas de fertilidad en la mujer. Una de las alternativas de elección para disminuir los efectos del SOP son las medidas higiénico dietéticas debido a que se enfoca en los diferentes aspectos que comprende la enfermedad. 
- Fomentar actividades comunitarias y sociales

- Brindar ayuda para reexaminar la percepsion de si mismo

- Ayudar a reconocer los sentimientos de tristeza y ansiedad por medio de conversaciones

- Ayudar a recococer de un ambiente agradable

- Ayudar a reconocer el ambiente obesogénico

El síndrome de ovario poliquístico es polimorfo y varia en sus características dependiendo el fenotipo; aproximadamente en el $70 \%$ de las mujeres predomina el fenotipo clásico y en menor cantidad el normo androgénico (Alicia et al. 2017). En el síndrome de ovario poliquístico se encuentran elevados los andrógenos en especial la testosterona, la cual es una hormona presente en todas las personas. Sin embargo, en la mujer, los niveles altos de esta hormona suelen producir infertilidad, acné, alteraciones menstruales, crecimiento de vello especialmente en cara; con la implementación de las medidas higiénico-dietéticas y el uso de metformina se logra disminuir los niveles de dicha hormona, reduciendo significativamente los síntomas que produce (Alicia et al. 2017). En el caso de la dieta, como se mencionó anteriormente, se basa en cambiar el tipo de alimento, con el objetivo de disminuir el consumo de calorías y aumentando la ingesta de alimentos ricos en fibra y cereales que ayudan a mantener los niveles normales de glucosa en sangre. La persona que lleva una buena alimentación suele tener una mejor calidad de vida e influye en gran medida en el control de las enfermedades. Las medidas higiénico dietéticas son la piedra angular para tener una vida libre de riesgos y enfermedades.

De acuerdo con Gaullo-Paca (Guallo-Paca 2020), en las mujeres con SOP está indicado 3 tipos de dieta, dirigidas a satisfacer las necesidades nutricionales, regular los niveles de insulina y mantener un peso saludable:

1. Dieta antiinflamatoria: EL consumo de pescado, frutas, verduras, aceite de oliva y hortalizas ayuda a reducir la respuesta inflamatoria por enfermedades de alta incidencia como, obesidad, diabetes, cáncer y enfermedades cardiovasculares.

2. Dieta hipocalórica: La preparación de los alimentos al vapor, horno, asada y hervida, reduce el valor calórico, logrando un balance energético y reduciendo el peso corporal. Por lo mismo, se debe evitar los alimentos fritos o con alto contenido graso - calórico como la margarina, aceite, quesos, bebidas alcohólicas y refrescos.

3. Dieta DASH (Acciones dietética para frenar hipertensión): Consiste en reducir el consumo de sal y alimentos que lo contengan, aumentar la ingesta de frutas y verduras, así como disminuir las grasas saturas y azucares (Ortega Anta et al. 2016).

\section{Tabla 3. Intervenciones de enfermería}

\begin{tabular}{|l|} 
Intervenciones de enfermeria \\
Primer nivel, promoción / prevención \\
Orientar al paciente y familiar sobre consultar \\
al ginecólogo en caso de: \\
- Periodos menstruales irregulares \\
- Presencia de acné \\
- Realizar deporte \\
- Ingerir una dieta balanceada \\
Segundo/tercer nivel \\
- Valorar el estado físico y emocional \\
- Revisar niveles hormonales y glucosa \\
- Tomar exámenes de laboratorio y \\
gabinete \\
Rehabilitación \\
- Importancia de acudir a los controles \\
- Mon el especialista \\
- Mantener la adherencia al \\
- tratamiento médico. \\
L Llevar a cabo una dieta equilibrada
\end{tabular}

Las mujeres que logran disminuir el peso corporal tienden a reducir las alteraciones metabólicas (diabetes mellitus tipo 2, resistencia a la insulina), mejoran su estado de ánimo debido a los cambios que presenta el cuerpo, logran asumir las problemáticas de la enfermedad con determinación y fortaleza. Los problemas psicológicos son un tema importante en la mujer con SOP debido a que sienten una pérdida de la identidad femenina a causa del acné, obesidad, hirsutismo, infertilidad y otros problemas que llegan a presentar. La mujer con síndrome de ovario poliquístico suele sufrir de depresión y ansiedad, ya que sienten perder la identidad por los problemas reproductivos (infertilidad) y apariencia física, afectando significativamente el estado emocional y mental de la mujer. La intervención multidisciplinaria ha demostrado ser más efectiva en la implementación de esta terapia y los profesionales de enfermería brindan atención a los diferentes aspectos ya mencionados por medio de intervenciones de enfermería mejorando el estado de la persona (Guallo-Paca 2020); (Geranne Jiskoot et al. 2020). Estas acciones son una alternativa eficaz para el tratamiento del síndrome de ovario poliquístico; la implementación de este tratamiento a largo plazo ayuda a reducir a tal grado los síntomas que la paciente puede llegar a no cumplir con los criterios del fenotipo de diagnóstico inicial, posicionarse en uno diferente y mejorar el estado general y emocional. Estudios revelan que en su mayoría las mujeres sometidas a este tratamiento logran disminuir su peso, reducción del tamaño del ovario y mejora de su estado psicológico (Alicia et al. 2017); (Geranne Jiskoot et al. 2020). De acuerdo a un estudio en 186 pacientes alrededor del $80.2 \%$ presento sobrepeso y obesidad, además, se observó que en el $30 \%$ de las mujeres refirieron la 
ingesta diaria de alimentos ricos en grasas. La implementación de las dietas antes mencionadas mostró mejoría en un $71.2 \%$ a la resistencia a la insulina debido al bajo consumo de alimentos grasos presentando sensibilidad a la insulina, mejorando alteraciones menstruales, así como disminución del peso corporal (Zheng et al. 2021); otros estudios demuestran que el $63 \%$ de 24 pacientes lograron mejoras mediante la implementación de las medidas higiénico dietéticas al notar, reducción de peso corporal, disminución de la glucosa, colesterol y triglicérido, mediante dietas y ejercicio (Szczuko, Malarczyk, and Zapałowska-Chwyć 2017); además en la utilización de la metformina, se observó en 97 pacientes, reducción del volumen ovárico, aumento de los ciclos menstruales así como reducción de los niveles de andrógenos disminuyendo el hirsutismo, acné y testosterona en el periodo de 6 meses (Sathyapalan et al. 2017). Los planes de cuidados enfermeros has sido empleados en distintos países, uno de ellos es Estado Unidos, considerándolo como base fundamental para la atención mediante intervenciones y actividades de enfermería, enfocadas en los distintos puntos que abarcan esta patología, iniciando con una serie de preguntas en apoyo con diversas escalas, como es la escala de estrés, ansiedad y depresión (DASS21). Recordando que el SOP afecta el estado psicológico del individuo (Tiara Dewi, Muhammad Amir Masruhim 2016), es de suma importancia la evaluación de depresión y/o ansiedad por personal de salud, para posteriormente intervenir en mejorar el estado de ánimo y buscar una mejor adherencia a las distintas intervenciones que se realicen en el paciente. Algunas recomendaciones incluyen (Teede et al. 2011):

- Dieta equilibrada que incluya un índice para prevenir aumento de peso y pérdida de peso en mujeres con sobrepeso.

- Asesoramiento dietético, que incluye educación y apoyo a mujeres con síndrome de ovario poliquístico.
Actualmente, algunos países están dirigiendo estas medidas hacia el profesional de enfermería. El Salvador extendió una guía para proporcionar información al profesional de enfermería sobre el síndrome de ovario poliquístico en los diferentes niveles de atención (Ministerio de Salud del Salvador 2019) (Tabla 3). Por otra parte, Ecuador cuenta con un plan de cuidados enfocados a las diferentes problemáticas previamente mencionadas (Sanchez Rivera 2018) (Tabla 4). Sin embargo, México no cuenta con estudios que demuestren la importancia de las medidas higiénico dietéticas por el profesional de enfermería y no posee programas específicos para este padecimiento. Por otra parte, cuenta con diversas estrategias que si bien no están enfocadas a este padecimiento atacan a los diversos aspectos que engloba el SOP. Un ejemplo de ello es la, Estrategia de educación nutricional y su importancia para combatir el sobrepeso y obesidad, en la cual se orienta a la población con información básica y valida respecto a una dieta correcta, accesible y saludable; promueve el consumo saludable de alimentos naturales que se encuentran en la canasta básica, mediante la implementación del semáforo de la nutrición indicando el valor nutritivo de cada alimento, incluyendo la NOM-051-SCFI/SSA1-2010 que consiste en sellos en el etiquetado de productos que rebasan los limites sobre calorías, sodio, entre otros (Secretaría de salud 2020). México no cuenta con planes de cuidados enfocados al síndrome de ovario poliquístico, sin embargo tiene planes encaminados a atender los diferentes aspectos del SOP (secreraria de salud 2016)(Tabla 5).

Otras actividades implementadas son:

- Conocer los gustos alimenticios del paciente, así como su estilo de vida para individualizar la dieta

- Discutir las necesidades nutricionales y la percepción del paciente de la dieta recomendada

- Planificar un programa de ejercicios

- Valorar el progreso

\section{Tabla 4. Plan de cuidados de enfermería en Ecuador}

\begin{tabular}{|c|c|c|c|}
\hline Diagnostico & Resultado & Intervenciones & Actividades \\
\hline $\begin{array}{l}00168 \text { - Estilo de } \\
\text { vida sedentario r/c } \\
\text { conocimientos }\end{array}$ & (2000) Calidad de vida & $\begin{array}{l}\text { (1030) Manejo de } \\
\text { los trastomos de la } \\
\text { alimentación }\end{array}$ & $\begin{array}{l}\text { Fomentar una alimentación } \\
\text { saludable }\end{array}$ \\
\hline $\begin{array}{l}00193 \text { - Descuido } \\
\text { personal r/c estrés }\end{array}$ & $\begin{array}{l}\text { (2006) Estado de salud } \\
\text { personal }\end{array}$ & $\begin{array}{l}\text { ( } 4350) \text { Manejo de la } \\
\text { conducta }\end{array}$ & $\begin{array}{l}\text { Planificar actividades de vida } \\
\text { diaria encaminadas a } \\
\text { disminuir el estrés }\end{array}$ \\
\hline $\begin{array}{l}00163 \\
\text { Disposición para } \\
\text { mejorar la nutrición } \\
\text { r/c consumo de } \\
\text { alimentos } \\
\text { inadecuados. }\end{array}$ & $\begin{array}{l}\text { (1009) Estado } \\
\text { nutricional: ingestión de } \\
\text { nutrientes }\end{array}$ & $\begin{array}{l}\text { (1100) Manejo de la } \\
\text { nutrición }\end{array}$ & $\begin{array}{l}\text { Proporcionar la información a } \\
\text { adecuada a la paciente para } \\
\text { que consuma alimentos que } \\
\text { le favorezca su nutrición }\end{array}$ \\
\hline
\end{tabular}




\section{Tabla 5. Plan de cuidados de enfermería en México}

\begin{tabular}{|c|c|c|c|}
\hline Diagnostico & Resultado & Intervención & Actividades \\
\hline $\begin{array}{l}\text { O0001- } \\
\text { Desequilibrio } \\
\text { Nutricional: por } \\
\text { exceso }\end{array}$ & $\begin{array}{l}(1612) \text { Control de } \\
\text { peso } \\
\text { (1008) Estado } \\
\text { nutricional: }\end{array}$ & $\begin{array}{l}\text { (1100) Manejo de la } \\
\text { Nutrición } \\
(1120) \text { Terapia nutricional } \\
\text { ( } 5614) \text { Ensefianza: dieta } \\
\text { prescrita }\end{array}$ & $\begin{array}{l}\text {-Determinar los hábitos } \\
\text { alimenticios e ingesta del paciente } \\
\text { y deseo para reducir el peso } \\
\text { - Establecer metas para el cambio } \\
\text { de conductas alimentarias y } \\
\text { pérdida de peso plan de comidas } \\
\text {-Desarrollar un plan dieta equilibrada } \\
\text { diarias con una dieta } \\
\text { baja en grasas y calorias }\end{array}$ \\
\hline $\begin{array}{l}00120 \text { - Baja } \\
\text { autoestima } \\
\text { situacional }\end{array}$ & $\begin{array}{l}\text { (1205) } \\
\text { Autoestima }\end{array}$ & $\begin{array}{l}\text { (5400) Potenciación de la } \\
\text { autoestima }\end{array}$ & $\begin{array}{l}\text {-Mostrar confianza en la } \\
\text { capacidad del paciente para } \\
\text { controlar una situación } \\
\text {-Identificar los factores estresantes } \\
\text { percibidos }\end{array}$ \\
\hline $\begin{array}{l}00146 \\
\text { Ansiedad }\end{array}$ & $\begin{array}{l}(1402) \\
\text { Autocontrol de la } \\
\text { ansiedad }\end{array}$ & $\begin{array}{l}\text { (5820) Disminución de la } \\
\text { ansiedad }\end{array}$ & $\begin{array}{l}\text { Favorecer una respiración lenta, } \\
\text { profunda, intencionadamente }\end{array}$ \\
\hline $\begin{array}{l}00179-\text { Riesgo } \\
\text { de nivel de } \\
\text { glucemia } \\
\text { inestable }\end{array}$ & $\begin{array}{l}(2300) \text { Nivel de } \\
\text { glucemia }\end{array}$ & $\begin{array}{l}\text { (5246) Asesoramiento } \\
\text { nutricional }\end{array}$ & $\begin{array}{l}\text { Determinar la ingesta y los hábitos } \\
\text { alimentarios del paciente }\end{array}$ \\
\hline
\end{tabular}

Actualmente no hay datos estadísticos que demuestren los beneficios de las intervenciones de enfermería antes mencionadas en las mujeres con síndrome de ovario poliquístico, sin embargo, es de gran relevancia la implementación de estas medidas en los diferentes sectores de salud para orientar al paciente sobre las complicaciones o riesgos que trae consigo en caso de no brindar una atención oportuna, así como mantener un control en las pacientes que lo presentan. Es importante la implementación de estas intervenciones debido a las alteraciones no solo a nivel físico sino mental y social debido a que suelen presentar depresión, alteración de la percepción corporal, así como a perdida de la identidad. Hay que recordar que es un problema de salud pública por la cantidad de casos presentados (Carron 2016).

Las necesidades que implica el síndrome de ovario poliquístico incitan al profesional de enfermería a crear planes de cuidados específicos para atenderlo. Los planes de cuidados fungen como instrumento de cuidado del paciente, basado en evidencia mediante intervenciones y actividades que están centrados en conocimiento científico sólido. La creación ayuda a dar un mejor cuidado debido a que no solo atiende las necesidades físicas del usuario sino las sociales, emocionales y espirituales. De la misma manera garantiza un cuidado de calidad y brinda apoyo al profesional de enfermería en la práctica. Los planes de cuidados enfermeros orientan las actividades y ejecución de acciones, además posibilita la interacción de los familiares con el paciente, creando un equipo coordinado que pueda realizar un cuidado, garantizando la atención integral. El plan de cuidados también aborda el pensamiento crítico, así como decisiones para crear un plan, integrando conocimientos de enfermería, experiencias previas en el cuidado del paciente con síndrome de ovario poliquístico; el profesional de enfermería debe prever las necesidades que requiere esta población específica para asegurar la integración de elementos de pensamiento crítico clave. Debido a lo antes mencionado es importante la creación de planes de cuidados enfocados al SOP que permitan comunicar la situación del paciente, estrategias, resultados esperados, indicación, etc. dirigidos a brindar un cuidado de calidad (Limachi and Nuñez 2020).

\section{Conclusión}

El síndrome de ovario poliquístico es un trastorno que afecta a la mujer en la edad reproductiva. Los principales motivos de consulta médica en la mayoría de las mujeres son trastornos menstruales, aumento de peso e infertilidad. De acuerdo a los investigadores la implementación de las medidas higiénico dietéticas en mujeres con SOP brinda varios beneficios, entre los que se encuentran; disminución de los síntomas, comorbilidades, problemas hormonales, disminución del peso corporal, reducción de los niveles de insulina, mejora los problemas psicológicos y restauración de la ovulación. Las medidas higiénico-dietéticas no solo atienden los fenotipos del SOP existentes, también, resuelven los problemas psicológicos que presentan las mujeres al perder la identidad femenina debido al hirsutismo y calvicie en algunos casos. Así que las medidas higiénico-dietéticas son una alternativa de primera línea para las personas que presentan síndrome de ovario poliquístico y un área de oportunidad para la intervención del profesional de enfermería. 


\section{Glosario}

Hiperandrogenismo: Aumento anormal de uno o varios andrógenos en sangre, por ejemplo, testosterona, total o libre y androstenediona.

Hirsutismo: Crecimiento excesivo de pelo en zonas generalmente asociadas con maduración sexual masculinizante frecuentemente en cara, tórax, abdomen, línea alba bajo, muslos y glúteos.

Andrógenos: Hormonas esteroides producida en el testículo, corteza suprarrenal y ovario. Entre los principales se encuentra la androstenediona y testosterona.

Alopecia: Pérdida anormal del cabello.

Dislipidemias: Condición de alteración del metabolismo de los lípidos y lipoproteínas en sangre.

Parestesia: Sensación de hormigueo de presencia temporal, producida en brazos, manos, piernas o pies.

Virilización: Es un tipo de hirsutismo extremo vinculado a calvicie, aumento del clítoris, hipertrofia muscular y voz masculina.

\section{Referencias}

Alfaro Murillo, Gabriela, Bárbara Salas Ramírez, and Alexa Zúñiga Fallas. 2021. "Evaluación Del Síndrome de Ovario Poliquístico." Revista Medica Sinergia 6 (1): e635. https://doi.org/10.31434/rms.v6i1.635.

Alicia, González Reigada, Félix González González I, and Diosdado Coll Bujardón I. 2017. "Respuesta Terapéutica En Pacientes Con Síndrome de Ovarios Poliquísticos Therapeutic Response in Patients with Polycistic Ovary Syndrome" 21 (2): 191-201.

Balestrini, Freddy Febres. 2016. "Etiología Del Síndrome de Ovario Poliquístico" 76 (Supl 1): 5-9.

Calpa Pastas, Angela María, Geiman Andrés Santacruz Bolaños, Marisol Álvarez Bravo, Christian Alexander Zambrano Guerrero, Edith de Lourdes Hernández Narváez, and Sonia Maritza Matabanchoy Tulcan. 2019. "Promoción de Estilos de Vida Saludables: Estrategias y Escenarios." Hacia La Promoción de La Salud 24 (2): 139-55. https://doi.org/10.17151/hpsal.2019.24.2.11.

Carmona-Ruiz, Israel Obed, Eric Saucedo-de la Llata, María Rosa Moraga-Sánchez, and Alberto Romeu-Sarrió. 2015. "Síndrome de Ovario Poliquístico: ¿Ha Aumentado Su Prevalencia?” Ginecologia y Obstetricia de Mexico 83 (12): 750-59.

Carron, Rebecca. 2016. "Update on Polycystic Ovary Syndrome: What Dermatology Nurses and Nurse Practitioners Need to Know." Journal of the Dermatology Nurses' Association 8 (6): 380-85. https://doi.org/10.1097/JDN.0000000000000259.

Chiliquinga Villacis, Sixto, Roberto Aguirre Fernández, Maritza Agudo
Gonzabay, Ángel Chú Lee, and Sylvana Cuenca Buele. 2017. "Criterios Diagnósticos y Tratamiento Integral Terapéutico Del Síndrome de Ovarios Poliquísticos." Revista Cubana de Obstetricia y Ginecologia 43 (3): 173-81.

Esparza-Romero, Julián. 2021. "Prevalencia y Factores Asociados a Diabetes Mellitus Tipo 2 En Población Indígena de México: Revisión Sistemática." Revista M dica Del Instituto Mexicano Del Seguro Social 58 (3): 1-9.

https://doi.org/10.24875/rmimss.m20000036.

Gómez Cruz, Zoila la, Patricia ia Landeros Ramírez, Enrique ue Romero Velarde, and Rogelio io Troyo Sanromán. 2016. "Estilos De Vida Y Riesgos Para La Salud En Una Población Universitaria." RESPYN Revista Salud Pública y Nutrición 15 (2): 15-21.

González, José Antonio, Santiago Echevarría, Leticia Aguilar, and Javier Davila. 2010. "Guía de Práctica Clínica Del Síndrome de Ovarios Poliquísticos, Evidencias y Recomendaciones." Instituto Mexicano Del Seguro Social, 50.

http://www.imss.gob.mx/sites/all/statics/guiasclinicas/000GER_Ov ariosPoliquisticos.pdf.

Guallo-Paca, Mariana. 2020. "Alimentación En Adolescentes Con Síndrome de Ovario Poliquístico." Polo Del Conocimiento 5 (07): 3-17. https://dialnet.unirioja.es/servlet/articulo?codigo=7518062.

Huérfano, Tanit, and María Ortiz. 2016. "Tratamiento No Farmacológico Del Síndrome de Ovario Poliquístico: Modificación Del Estilo de Vida: Ejercicio y Nutrición.” Revista de Obstetricia y Ginecología de Venezuela, no. Volume 76

Jiskoot, G., S. H. Benneheij, A. Beerthuizen, J. E. De Niet, C. De Klerk, R. Timman, J. J. Busschbach, and J. S.E. Laven. 2017. "A ThreeComponent Cognitive Behavioural Lifestyle Program for Preconceptional Weight-Loss in Women with Polycystic Ovary Syndrome (PCOS): A Protocol for a Randomized Controlled Trial." Reproductive Health 14 (1): 1-12. https://doi.org/10.1186/s12978-017-0295-4

Jiskoot, Geranne, Alexandra Dietz de Loos, Annemerle Beerthuizen, Reinier Timman, Jan Busschbach, and Joop Laven. 2020. "LongTerm Effects of a Three-Component Lifestyle Intervention on Emotional Well-Being in Women with Polycystic Ovary Syndrome (PCOS): A Secondary Analysis of a Randomized Controlled Trial." PLOS ONE 15 (6): 1-16. https://doi.org/10.1371/journal.pone.0233876

Limachi, Miranda, and Rodriguez Nuñez. 2020. "Proceso de Atencion de Enfermeria Como Instrumento Del Cuidado, Significado Para Estudiantes de Ultimo Curso," 374-89. https://doi.org/https://doi.org/10.22201/eneo.23958421e.2019.4.62 3.

Luis Higuera-Sainz, José, ; José, Alfredo Pimentel-Jaimes, ; Geu, Salomé Mendoza-Catalán, Ulises Rieke-Campoy, Gisela Ponce, Ponce De León, and Adriana Camargo-Bravo. 2017. "El Consumo De Alcohol Como Factor De Riesgo Para Adquirir Sobrepeso Y Obesidad Alcohol Consumption As a Risk Factor To Acquire Overweight and Obesity." Ra Ximhai, 53-62.

Ministerio de Salud del Salvador. 2019. "Guías de Atención de Enfermería En Ginecoobstetricia y Perinatología." Unidad Nacional de Enfermería, 1-169.

http://asp.salud.gob.sv/regulacion/pdf/guia/guias_de_atencion_de enfermeria_en_ginecoobstetricia_y_perinatologia_marzo2019.pdf.

Moncada, Silder Javier, Andrea Estefanía Ramos, and Luis Gerardo Muñoz. 2018. "Artículo Original Polycystic Ovary Syndrome: Confirmed Cases and Treatment in the Outpatient Clinic Of "Hospitalof"Hospital Escuela." Rev. Fac. Cienc. Méd. Julio- 
Diciembre, 17-23.

Monteagudo Peña, Gilda, Roberto González Suárez, Manuel Gómez Alzugaray, Gisel Ovies Carballo, Ahmed Menocal Alayón, Kenia Rodríguez Martínez, Jorge Puentes Corral, and Yaima Bell Hernández. 2019. "Resistencia a La Insulina En Mujeres Con Síndrome de Ovario Poliquístico.” Revista Cubana de Endocrinología 30 (2): 1-23.

Orrego, A. 2019. “Acercamiento Actualizado a La Fisiopatología, Clasificación y Genética Del Síndrome de Ovarios Poliquísticos." Revista Colombiana de Endocrinología, Diabetes \& Metabolismo 6 (2): 101-6. https://doi.org/10.53853/encr.6.2.484.

Ortega Anta, Rosa M., Ana Isabel Jiménez Ortega, José Miguel Perea Sánchez, Esther Cuadrado Soto, and Ana M. López-Sobaler. 2016. "Pautas Nutricionales En Prevención y Control de La Hipertensión Arterial." Nutricion Hospitalaria 33: 53-58. https://doi.org/10.20960/nh.347.

Poliqu, Ovario. 2019. "Los Integrantes Del Comité Aprueban La Tesis de Frecuencia de Variantes Fenotípicas En Pacientes Con Síndrome de Ovario Poliquístico, Que Presenta Para Cubrir El Requisito Parcial de Obtención Del Grado de : Comité de Tesis."

Rivera, Nidia, Patricia Rodríguez, and Juan Guarneros. 2017. "Hipoglucemiantes Orales Para El Tratamiento de Diabetes Mellitus Tipo 2: Uso y Regulación En México." Rev Hosp Јиа Mex 84 (4): 203-11.

www.medigraphic.com/hospitaljuarezwww.medigraphic.org.mxw ww.medigraphic.org.mx.

SANCHEZ RIVERA, LEIDY ELENA. 2018. Unidad Académica de Ciencias Químicas y de La Salud Carrera de Enfermería. Revista Cubana de Enfermería. Vol. 27. http://repositorio.utmachala.edu.ec/bitstream/48000/10790/1/CHU CHUCA CAIMINAGUA MARITZA JACQUELINE.pdf.

Sathyapalan, Thozhukat, Anne Marie Coady, Eric S. Kilpatrick, and Stephen L. Atkin. 2017. "The Effect of Atorvastatin on Pancreatic Beta Cell Requirement in Women with Polycystic Ovary Syndrome." Endocrine Connections 6 (8): 811-16. https://doi.org/10.1530/EC-17-0217.

secreraria de salud. 2016. "Plan de Cuidados de Enfermeria Estandarizados," $1-73$. https://ssj.jalisco.gob.mx/sites/ssj.jalisco.gob.mx/files/place_obesid ad.pdf.

Secretaría de salud. 2020. "Estrategia de Educación Nutricional y Su Importancia Para Combatir El Sobrepeso y Obesidad En México." 2020. https://www.gob.mx/agricultura/articulos/estrategia-deeducacion-nutricional-y-su-importancia-para-combatir-elsobrepeso-y-obesidad-en-mexico?idiom=es.

Suarez Coba, Becquer Humberto, Patricia Elisabeth Borja Tapia, Mayra Alexandra Vela Chasiluisa, and Carlos Fabián Ontaneda Tenesaca. 2019. "Diagnóstico y Manejo Del Síndrome de Ovario Poliquístico." Recimundo 3 (3): 970-1004. https://doi.org/10.26820/recimundo/3.(3).septiembre.2019.9701004.

Szczuko, Małgorzata, Inez Malarczyk, and Marta Zapałowska-Chwyć 2017. "Improvement in Anthropometric Parameters after Rational Dietary Intervention in Women with Polycystic Ovary Syndrom as the Best Method to Support Treatment." Roczniki Panstwowego Zakladu Higieny 68 (4): 409-17.

Teede, Helena J., Marie L. Misso, Amanda A. Deeks, Lisa J. Moran, Bronwyn G.A. Stuckey, Jennifer L.A. Wong, Robert J. Norman, and Michael F. Costello. 2011. "Assessment and Management of
Polycystic Ovary Syndrome: Summary of an Evidence-Based Guideline." Medical Journal of Australia 195 (6): S65-112. https://doi.org/10.5694/mja11.10915.

Tiara Dewi, Muhammad Amir Masruhim, Riski Sulistiarini. 2016. "Uso de Las Escalas de Depresión Ansiedad Estrés (DASS-21) Como Instrumento de Tamizaje En Jóvenes Con Problemas Clínicos." Laboratorium Penelitian Dan Pengembangan FARMAKA TROPIS Fakultas Farmasi Universitas Mualawarman, Samarinda, Kalimantan Timur 6 (April): 5-24.

Velázquez, Elsy. 2016. "Sensibilizadores a La Insulina." Revista de Obstetricia y Ginecología de Venezuela 76 (Volume 76).

Vidilia, Jaylin, Carmenate Pérez, Eduardo Cabrera Rode, Gilda Monteagudo Peña, and Amalia Peix González. 2019. “Asociación Entre El Fenotipo Hipertrigliceridemia-Obesidad Abdominal , La Resistencia a La Insulina y Las Variables Cardiometabólicas En E Síndrome de Ovario Poliquístico Association among the Hypertriglyceridemic Waist Phenotype , Insulin Resistance And." Revista Cubana de Endrocrinología 2 (30): 1-20.

Zheng, Xin, Yun Chen, Danyan Ma, Mulin Zhang, Yinxiang Huang, Meifeng Tong, Bing Yan, Shaowei Lin, Xiaohong Yan, and Changqin Liu. 2021. "Correlation between Daily Energy Intake from Fat with Insulin Resistance in Patients with Polycystic Ovary Syndrome." Diabetes, Metabolic Syndrome and Obesity: Targets and Therapy 14: 295-303. https://doi.org/10.2147/DMSO.S287936. 